\title{
Phase Transitions of Thermoelectric TAGS-85
}

\author{
Anil Kumar, ${ }^{\dagger}$ Paul A. Vermeulen, ${ }^{\dagger}$ B Bart J. Kooi, ${ }^{\dagger}$ Jiancun Rao, $^{\ddagger}, \perp$ Lambert van Eijck, \\ Stefan Schwarzmüller," Oliver Oeckler, ${ }^{\| \odot}$ and Graeme R. Blake*,†
}

${ }^{\dagger}$ Zernike Institute for Advanced Materials, University of Groningen, Nijenborgh 4, 9747AG Groningen, The Netherlands

${ }^{\ddagger}$ School of Materials Science and Engineering, Harbin Institute of Technology, 150001 Harbin, P. R. China

${ }^{\S}$ Department of Radiation Science and Technology, Delft University of Technology, Mekelweg 15, 2629JB Delft, The Netherlands

"Institute for Mineralogy, Crystallography and Materials Science, Leipzig University, Scharnhorststrasse 20, 04275 Leipzig, Germany

${ }^{\perp}$ AIM Lab, Maryland NanoCenter, University of Maryland, College Park, Maryland 20742, United States

\section{Supporting Information}

ABSTRACT: The alloys $\left(\mathrm{GeTe}_{x}\left(\mathrm{AgSbTe}_{2}\right)_{100-x}\right.$, commonly known as TAGS- $x$, are among the best performing p-type thermoelectric materials for the composition range $80 \leq x \leq 90$ and in the temperature range $200-500{ }^{\circ} \mathrm{C}$. They adopt a rhombohedrally distorted rocksalt structure at room temperature and are reported to undergo a reversible phase transition to a cubic structure at $\sim 250{ }^{\circ} \mathrm{C}$. However, we show that, for the optimal $x=85$ composition (TAGS-85), both the structural and thermoelectric properties are highly sensitive to the initial synthesis method employed. Single-phase rhombohedral samples exhibit the best thermoelectric properties but can only be obtained after an annealing step at $600{ }^{\circ} \mathrm{C}$ during initial cooling from the melt. Under faster cooling conditions, the samples obtained are

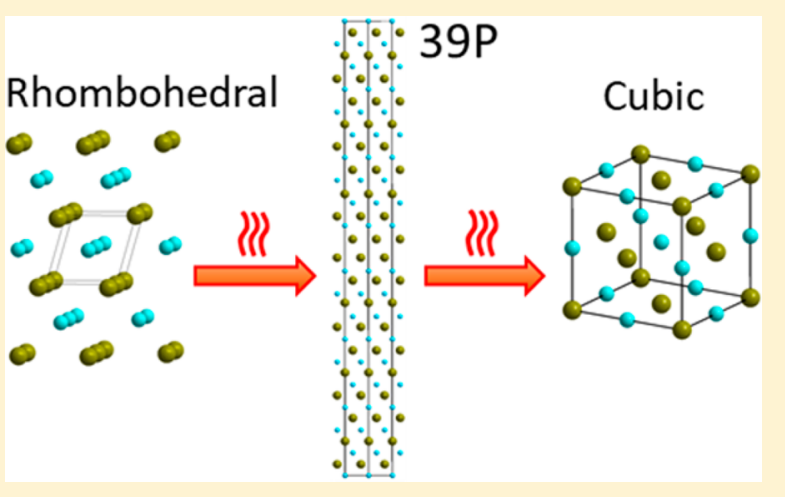
inhomogeneous, containing multiple rhombohedral phases with a range of lattice parameters and exhibiting inferior thermoelectric properties. We also find that when the room-temperature rhombohedral phase is heated, an intermediate trigonal structure containing ordered cation vacancy layers is formed at $\sim 200{ }^{\circ} \mathrm{C}$, driven by the spontaneous precipitation of argyroditetype $\mathrm{Ag}_{8} \mathrm{GeTe}_{6}$ which alters the stoichiometry of the TAGS-85 matrix. The rhombohedral and trigonal phases of TAGS-85 coexist up to $380{ }^{\circ} \mathrm{C}$, above which a single cubic phase is obtained and the $\mathrm{Ag}_{8} \mathrm{GeTe}_{6}$ precipitates redissolve into the matrix. On subsequent cooling a mixture of rhombohedral, trigonal, and $\mathrm{Ag}_{8} \mathrm{GeTe}_{6}$ phases is again obtained. Initially single-phase samples exhibit thermoelectric power factors of up to $0.0035 \mathrm{~W} \mathrm{~m}^{-1} \mathrm{~K}^{-2}$ at $500{ }^{\circ} \mathrm{C}$, a value that is maintained on subsequent thermal cycling and which represents the highest power factor yet reported for undoped TAGS-85. Therefore, control over the structural homogeneity of TAGS-85 as demonstrated here is essential in order to optimize the thermoelectric performance.

\section{INTRODUCTION}

Thermoelectric materials are currently attracting much attention due to their potential for the conversion of thermal energy such as waste heat to electrical power, as well as their application in solid-state cooling systems. ${ }^{1-4}$ Although they are commercially produced on a small scale, the energy conversion efficiency of available materials is still too low to be costeffective for widespread applications. Thermoelectric performance is usually quantified by the so-called figure of merit $\mathrm{ZT}=$ $S^{2} \sigma T / \kappa$ and is maximized for a high Seebeck coefficient $(S)$ and electrical conductivity $(\sigma)$ and low thermal conductivity $(\kappa)$. Chalcogenide materials are among the best thermoelectrics for operation up to $\sim 600{ }^{\circ} \mathrm{C}$. In particular, despite the scarcity and relatively high cost of tellurium, materials based on GeTe have attracted much interest due to their good performance and chemical/mechanical stability in the $200-500{ }^{\circ} \mathrm{C}$ range, leading to high device reliability in heat harvesting applications. GeTe is a narrow band p-type semiconductor due to vacancies on the
Ge-substructure, each of which generates two holes. ${ }^{5}$ It exhibits a high thermoelectric power factor $S^{2} \sigma$, and the vacancies can also scatter phonons, reducing the lattice thermal conductivity. ${ }^{6,7}$ Moreover, GeTe and related materials are naturally nanostructured as a consequence of their crystal symmetry, spontaneously adopting a complex herringbone-like domain structure with domain width of a few hundred nanometers, ${ }^{8-10}$ which provides an additional phonon scattering mechanism.

Here we revisit a well-known thermoelectric material with composition $(\mathrm{GeTe})_{x}\left(\mathrm{AgSbTe}_{2}\right)_{100-x}$, which is commonly referred to as TAGS- $x$. Compositions in the range $75 \leq x \leq$ 90 exhibit much lower phononic thermal conductivity than the parent compound GeTe while maintaining high power factors due to a similar electronic structure with a highly degenerate Fermi surface. ${ }^{11}$ TAGS- 80 and TAGS-85 are of the most

Received: September 22, 2017

Published: November 29, 2017 

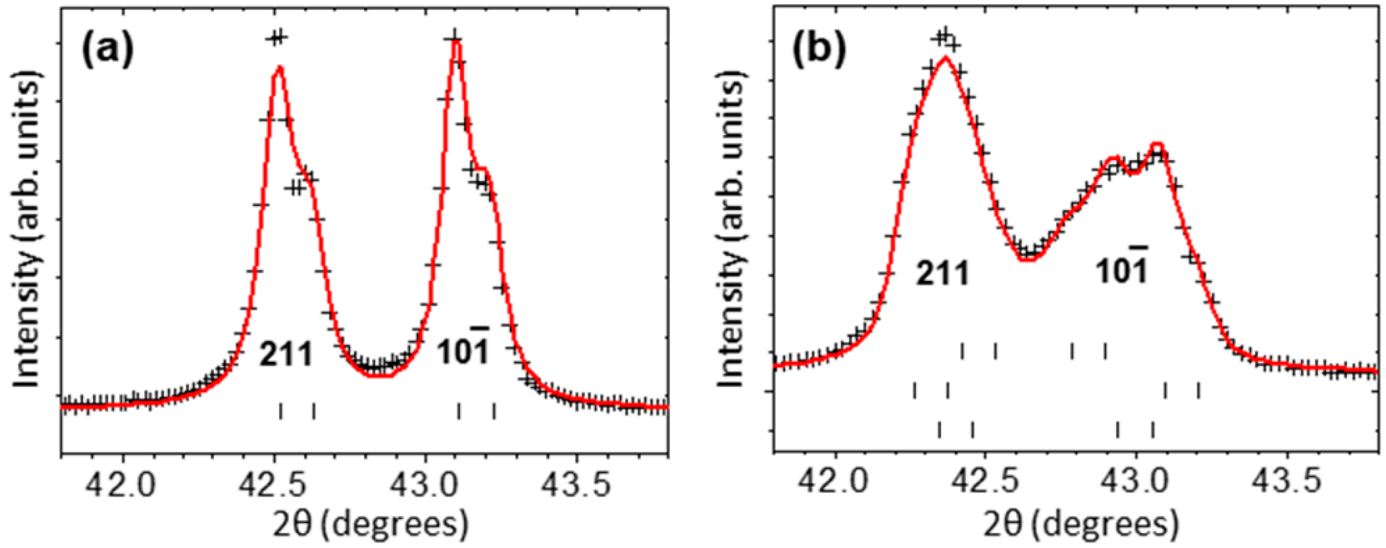

Figure 1. Partial room-temperature XRD patterns showing the region between $2 \theta=41.8^{\circ}$ and $43.8^{\circ}$ for TAGS-85 samples synthesized using different cooling methods. (a) Intermediate annealing at $600^{\circ} \mathrm{C}$ for $3 \mathrm{~h}$ gives a single rhombohedral phase. (b) Omitting the intermediate annealing step gives a multiphase sample; the XRD pattern was fitted using three rhombohedral phases with slightly different lattice parameters. Black data points and red curves represent the measured and fitted diffraction patterns, respectively. The lower tick marks indicate calculated peak positions.

interest, and $\mathrm{ZT}$ values of up to 1.75 at $500{ }^{\circ} \mathrm{C}$ have been reported for TAGS-80. ${ }^{12}$ However, TAGS- 85 is mechanically more stable ${ }^{13}$ and hence more suitable for applications despite its lower ZT of $\sim 1.2-1.4$ at the same temperature. ${ }^{12}$ Recently, the ZT value of TAGS-85 has been improved to the 1.5-1.6 range by doping with $\mathrm{Ce}$ or $\mathrm{Dy},{ }^{14,15}$ by intentionally introducing a high concentration of cation vacancies, ${ }^{16}$ or by artificial nanostructuring. ${ }^{17}$ The first two approaches increase the power factor whereas the latter decreases $\kappa$ without degrading the power factor because the carrier mean-free path is on the order of only $10 \AA$.

Nevertheless, when reviewing the literature it is noticeable that the measured thermoelectric properties of TAGS-85 (S, $\sigma$, and $\kappa$ ) vary significantly among different reports (Supporting Information, Table S1). Although differing measurement procedures and equipment can certainly contribute to this variability, it is worth considering whether exactly the same material has been studied in each case. Synthesis conditions vary among reports, ranging from direct quenching from the melt, ${ }^{14,15}$ holding at an intermediate temperature on initial cooling, ${ }^{18,19}$ subsequent annealing after cooling to room temperature, ${ }^{13}$ or additional grinding and hot-pressing steps. ${ }^{12,17,20}$ Furthermore, recent papers have not closely examined the crystal structure of TAGS-85, the sample homogeneity in terms of structure, or the possible dependence of these aspects on synthesis conditions, because there is generally assumed to be a well-characterized, smooth transition between the $\mathrm{R}$ - and $\mathrm{C}$-phases taking place at a temperature between 150 and $300{ }^{\circ} \mathrm{C}$ depending on the study. ${ }^{12,18,19}$ However, in some cases where X-ray diffraction data are presented, close inspection reveals irregular or asymmetric peak shapes suggesting inhomogeneous samples, ${ }^{12,14,15,21}$ or weak extra reflections implying the presence of impurity phases. ${ }^{20}$

Since it can be expected that the thermoelectric properties of TAGS-85 sensitively depend on details of the crystal structure, it is of interest to examine more closely the influence of the chemical synthesis procedure, as well as how the structural and thermoelectric properties evolve with repeated thermal cycling over the operating temperature range.

Here we show that careful control of the synthesis conditions is necessary to obtain single-phase rhombohedral samples at room temperature. Such samples exhibit a record high thermoelectric power factor for undoped TAGS-85, a perform- ance that is robust with respect to thermal cycling up to $500^{\circ} \mathrm{C}$. Furthermore, we show that the rhombohedral phase is not directly transformed to the cubic phase on heating as previously assumed, but rather it coexists with both an intermediate trigonal structure containing ordered cation vacancy layers and with micron-sized precipitates of argyrodite-type $\mathrm{Ag}_{8} \mathrm{GeTe}_{6}$ that redissolve in the TAGS- 85 matrix when the cubic phase is reached above $380{ }^{\circ} \mathrm{C}$.

\section{EXPERIMENTAL SECTION}

Stoichiometric amounts of the elements $\mathrm{Ge}, \mathrm{Te}, \mathrm{Ag}$, and $\mathrm{Sb}$ (purity 99.99\%) were weighed and mixed together using a mortar and pestle. The mixture was placed in a quartz ampule, which was evacuated to $10^{-2}-10^{-3}$ Torr $(1.33-0.133 \mathrm{~Pa})$ and sealed. The sealed ampule was placed horizontally in a tubular furnace and heated to $850{ }^{\circ} \mathrm{C}$ for $1 \mathrm{~h}$, at which temperature the mixture is molten, and the ampule was rocked and rotated every $10 \mathrm{~min}$ in order to ensure good homogeneity. The melt was then cooled down to $600{ }^{\circ} \mathrm{C}$ over $4 \mathrm{~h}$ and annealed for various lengths of time at constant temperature before quenching to room temperature in water. Solidified shiny ingots were obtained with an approximately rectangular shape and dimensions that varied within the range $15-20 \mathrm{~mm} \times 12-14 \mathrm{~mm}$ $\times 3-4 \mathrm{~mm}$. The ingots were sliced to appropriate dimensions using a diamond wire saw and then polished to obtain samples with flat surfaces and uniform thickness for high-temperature thermoelectric measurements.

High-temperature X-ray powder diffraction (XRD) patterns were recorded on crushed ingots using a Bruker D8 diffractometer operating in Bragg-Brentano geometry with $\mathrm{Cu} \mathrm{K} \alpha$ radiation and combined with an Anton Paar TTK-450 hot stage. The sample chamber was evacuated to $\sim 10^{-3}$ mbar $(\sim 0.1 \mathrm{~Pa})$. The sample temperature was controlled with a TCU-100 temperature control unit to a precision of within $\pm 1{ }^{\circ} \mathrm{C}$. The sample was heated or cooled at a rate of $0.5^{\circ} \mathrm{C} \mathrm{s}^{-1}$ to the set-point temperature, at which it was held for $300 \mathrm{~s}$ before measurement in order to ensure thermal equilibrium. Low-temperature powder XRD patterns were recorded with a Huber G670 diffractometer combined with a closed-cycle refrigerator and operating in the Guinier geometry with $\mathrm{Cu} \mathrm{K} \alpha_{1}$ radiation. The sample chamber was evacuated to $\sim 10^{-3}$ mbar $(\sim 0.1 \mathrm{~Pa})$. A roomtemperature neutron diffraction pattern (wavelength $1.667 \AA$ ) was collected on one sample of mass $5 \mathrm{~g}$ at the PEARL beamline at the Reactor Institute Delft. ${ }^{22}$ All data were analyzed using the GSAS software. $^{23}$

High-temperature Seebeck coefficient and electrical conductivity measurements were performed simultaneously using a Linseis LSR-3 apparatus. Thermal diffusivity $\left(D_{\mathrm{t}}\right)$ was measured by the laser flash method using a Linseis LFA1000 apparatus equipped with an InSb 

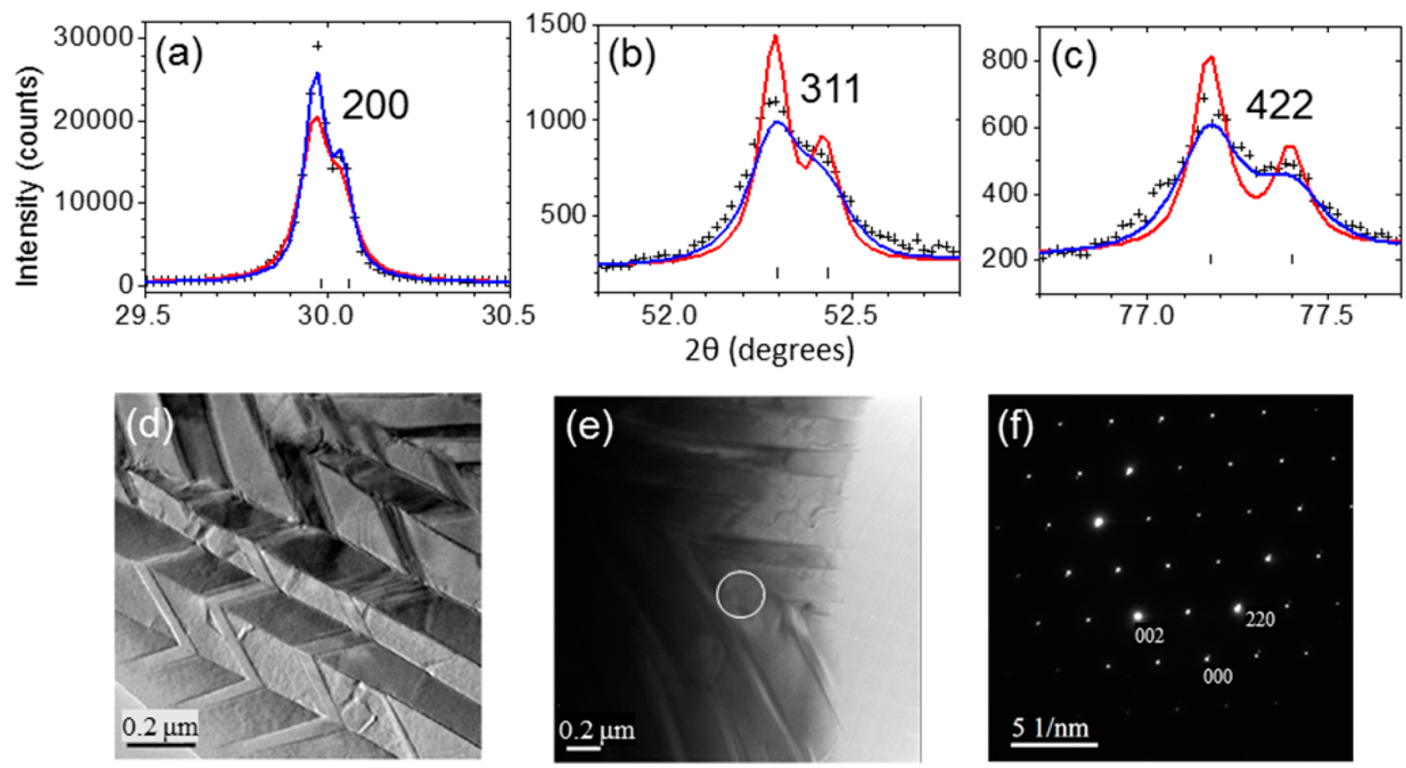

Figure 2. Structural properties of the R-phase of TAGS-85. (a-c) Selected XRD peaks of an as-synthesized, single-phase sample fitted without (red curve) and with (blue curve) the anisotropic microstrain broadening model. (d, e) Bright-field TEM images for the as-synthesized R-phase. (f) [11̄0] zone axis SAED pattern obtained from the single-domain, V-shaped area marked by the white circle in part e. In order to more easily relate the XRD peak widths to the nanodomain structure, all Miller indices refer to the cubic rocksalt unit cell.

detector in a He atmosphere from 300 to $773 \mathrm{~K}$. Up to five data points of $D_{\mathrm{t}}$ were merged after evaluating the quality of the fitted model $^{24}$ and excluding outliers at each temperature step of $50{ }^{\circ} \mathrm{C}$, starting from 50 ${ }^{\circ} \mathrm{C}$ up to $500{ }^{\circ} \mathrm{C}$. Thermal conductivity was calculated using the formula $\kappa=D_{\mathrm{t}} \times d \times C_{\mathrm{p}}$. Here the density $d$ was calculated from the mass and volume of the sample determined by the Archimedes principle. The Dulong-Petit approximation was used for the specific heat capacity $C_{\mathrm{p}}$, which is known to be a good estimate for TAGS and other telluride materials, within the large experimental uncertainties that result from fitting baselines in $C_{\mathrm{p}}$ measurements. ${ }^{16,25}$ Calculated ZT values exhibit an uncertainty of $\sim 20 \%$.

The nanostructure of the samples was studied by transmission electron microscopy (TEM). Samples were sliced and glued inside brass tubes of $3 \mathrm{~mm}$ diameter and cut into TEM sample disks. The disks were then ground, dimpled, and ion milled using a Gatan PIPS II at $6^{\circ}$ with an accelerating voltage that was ramped from 4 to $0.2 \mathrm{kV}$ to achieve electron transparency. TEM images and electron diffraction patterns were obtained using JEM 2010 and JEM 2010F microscopes operated at $200 \mathrm{kV}$. The chemical compositions of the samples were investigated using energy-dispersive X-ray spectroscopy (EDX) in the TEM, using a $\mathrm{Si}(\mathrm{Li})$ detector. Cliff-Lorimer fitting without absorbance was performed with the NSS 2.3 software (Thermo Scientific) to obtain accurate composition information. In some cases, samples were prepared by a FIB (focused ion beam) technique. TEM images and electron diffraction patterns were then produced with a Tecnai G2 F30 S-Twin microscope at an accelerating voltage of 300 $\mathrm{kV}$.

\section{RESULTS AND DISCUSSION}

1. Synthesis and Room-Temperature Structure. The way in which a sample is initially cooled from the melt determines its purity at room temperature and also how its thermoelectric properties evolve with subsequent thermal cycling. We systematically tested many different cooling protocols and found that pure, homogeneous R-phase samples can only be obtained by holding the sample for $3 \mathrm{~h}$ or more at an intermediate temperature of $600{ }^{\circ} \mathrm{C}$ during the initial cooling procedure. The single-phase nature of such samples is evidenced by a well-defined doublet between $2 \theta=42^{\circ}$ and $44^{\circ}$ in the partial XRD pattern shown in Figure 1a (measured for a sample held for $3 \mathrm{~h}$ at $600{ }^{\circ} \mathrm{C}$ ). The splitting between these 211 and $10 \overline{1}$ reflections is proportional to the deviation of the rhombohedral unit cell angle from $60^{\circ}$ (which would correspond to the cubic rocksalt structure). We note that the individual peaks appear as doublets because their widths are close to the instrumental resolution, allowing splitting from the $\mathrm{Cu} \mathrm{K} \alpha_{1}$ and $\mathrm{K} \alpha_{2}$ components of the X-ray beam to be visible. The XRD pattern of this sample could be fitted using the structural model of rhombohedral GeTe with the space group $\mathrm{R} 3 \mathrm{~m}$. The $\mathrm{Ge} / \mathrm{Ag} / \mathrm{Sb}$ atoms occupy the 1 a Wyckoff position with fixed coordinates of $(0,0,0)$, and the Te atoms occupy the $1 a$ position with refined coordinates of $(x, x, x)$, where $x=(0.5$ $+\delta)$. The peak shapes were modeled using a pseudo-Voigt function, but on close inspection significant anisotropic broadening was observed. The addition of anisotropic microstrain broadening parameters following the Stephens model ${ }^{26}$ as incorporated in the GSAS software significantly improved the fit (wRp decreased from 0.139 to 0.106 ), but the shapes of some of the broader peaks remained imperfectly fitted (Figure $2 a-c)$. The chemical composition of the sample was determined by EDX analysis and is listed in Table S2 of the Supporting Information, showing that the composition does not deviate significantly from the nominal stoichiometry of TAGS-85.

The anisotropic XRD peak broadening is most likely a consequence of the herringbone twin domain pattern that is apparent in the bright-field TEM images in Figure 2d,e. Such domain structures have previously been observed both in GeTe and TAGS- $x$ and result from the cubic-rhombohedral transition. ${ }^{8,19,21,27,28}$ The rhombohedral distortion involves an elongation of the cubic rocksalt unit cell along any of the four $\langle 111\rangle$ directions due to coherent relative shifts of the $\mathrm{Ge} / \mathrm{Sb}$ / $\mathrm{Ag}$ and Te sublattices in this direction, often referred to as a Peierls distortion. ${ }^{29}$ The resulting strain is accommodated by formation of a high density of stripe-like twin domains with widths in our case of $\sim 50 \mathrm{~nm}$. Because the R-phase has polar symmetry, a pair of inversion twins is also formed for each of the four domains, corresponding to opposite relative 
substructure shifts along [111]. By analogy with GeTe, the twin boundaries comprising the herringbone pattern are likely to be $\{100\}$ and $\{110\}$ planes, where the $\{100\}$ boundaries tend to be longer. ${ }^{28}$ In our XRD pattern, the 200 and 400 reflections (referred to the pseudocubic rocksalt unit cell) are sharpest while the 311,222 , and 422 reflections are broadest (Figure $2 \mathrm{a}-\mathrm{c})$. This is consistent with a domain pattern where the longest structural coherence is parallel to the "long" $\{100\}$ domain walls. Figure $2 \mathrm{f}$ shows a $[1 \overline{1} 0]$ zone axis selected area diffraction (SAED) pattern obtained from the single-domain area marked by the white circle in Figure 2e. The measured angle between the cubic (002) and (220) planes is $\sim 88^{\circ}$, consistent with a distortion from cubic symmetry as observed by XRD.

An XRD pattern was also collected at $20 \mathrm{~K}$ and showed a slightly smaller unit cell angle (stronger rhombohedral distortion) but essentially the same pattern of peak broadening. The refined crystallographic data for the R-phase at both room temperature and $20 \mathrm{~K}$ are summarized in Table 1 , and the fitted room-temperature XRD profile is shown in the Supporting Information, Figure S1a.

Table 1. Crystallographic Data for Rietveld Refinements of TAGS-85 at 295 and $20 \mathrm{~K}$ (Space Group R3m (No. 160))

\begin{tabular}{|c|c|c|}
\hline & \multicolumn{2}{|c|}{ temperature } \\
\hline & $295 \mathrm{~K}$ & $20 \mathrm{~K}$ \\
\hline lattice params & $\begin{array}{l}a=4.26992(9) \AA \\
\alpha=59.125(1)^{\circ}\end{array}$ & $\begin{array}{l}a=4.26514(13) \AA \\
\alpha=58.846(1)^{\circ}\end{array}$ \\
\hline cell volume $\left(\AA^{3}\right)$ & $53.951(2)$ & $53.418(3)$ \\
\hline $\mathrm{X}$-ray density $\left(\mathrm{g} \mathrm{cm}^{-3}\right)$ & 6.500 & 6.565 \\
\hline $\mathrm{Ge} / \mathrm{Ag} / \mathrm{Sb}$ coordinates & $(0,0,0)$ & $(0,0,0)$ \\
\hline $\mathrm{Ge} / \mathrm{Sb} / \mathrm{Ag} U_{\text {iso }}\left(\AA^{2}\right)$ & $0.075(2)$ & $0.0494(13)$ \\
\hline Te coordinate $(x, x, x)$ & $0.5171(5)$ & $0.5192(5)$ \\
\hline $\operatorname{Te} U_{\text {iso }}\left(\AA^{2}\right)$ & $0.058(2)$ & $0.0168(6)$ \\
\hline \multirow{4}{*}{$\begin{array}{l}\text { Stephens anisotropic peak } \\
\text { broadening params }\end{array}$} & $S_{400}=1.07(9)$ & $S_{400}=2.61(8)$ \\
\hline & $S_{220}=1.47(8)$ & $S_{220}=4.52(9)$ \\
\hline & $S_{310}=-1.05(5)$ & $S_{310}=-3.05(4)$ \\
\hline & $S_{211}=0$ & $S_{211}=0$ \\
\hline wRp & 0.106 & 0.012 \\
\hline$R\left(F^{2}\right)$ & 0.0679 & 0.138 \\
\hline
\end{tabular}

Single-phase samples could not be obtained after holding at $600{ }^{\circ} \mathrm{C}$ for less than $3 \mathrm{~h}$ on initial cooling, or after holding at a lower intermediate temperature. In such cases, the powder XRD patterns of the resulting samples could only be satisfactorily fitted using multiple rhombohedral phases with slightly different lattice parameters. A typical partial XRD pattern is shown in Figure $1 \mathrm{~b}$ for a sample that was cooled from the melt to $500{ }^{\circ} \mathrm{C}$ over $4 \mathrm{~h}$ and then directly quenched, where a fit to the doublet of 211 and $10 \overline{1}$ reflections using three rhombohedral phases is shown. The full fitted XRD profile for this sample is shown in the Supporting Information, Figure S1b. The absence of a sufficient annealing step on initial cooling thus leads to inhomogeneous samples, perhaps with a distribution of different chemical compositions or vacancy concentrations across the ingot. It should be noted that in some literature studies of TAGS- 85 the samples were prepared by direct quenching from the liquid phase, ${ }^{14,15}$ and in several cases the XRD patterns are characteristic of samples with multiple phases or chemical composition gradients. ${ }^{12,14,15,21}$
2. High-Temperature Structural Properties. XRD data were collected while heating a single R-phase sample. New reflections appear at $200{ }^{\circ} \mathrm{C}$, indicating the growth of a second phase that coexists with the R-phase (Figure 3a). The intensities of the new peaks are largest between 260 and 320 ${ }^{\circ} \mathrm{C}$ before declining with further heating and disappearing above $380{ }^{\circ} \mathrm{C}$. The peaks of the new phase at $280{ }^{\circ} \mathrm{C}$ could be indexed in a trigonal unit cell with lattice parameters $a=$ 4.2333(7) $\AA, c=69.895(17) \AA$. Here we note that the R-phase of TAGS-85 can be described in an equivalent hexagonal unit cell in which six alternating $\mathrm{Te}$ and $\mathrm{Ge} / \mathrm{Ag} / \mathrm{Sb}$ layers are stacked along [001] (see Supporting Information, Figure S2). The corresponding hexagonal unit cell parameters at $280{ }^{\circ} \mathrm{C}$ are $a=4.2470(9) \AA, c=10.4735$ (9) $\AA$. The $c$-lattice parameter of the trigonal phase would then correspond to $\sim 40$ alternating $\mathrm{Te}$ and $\mathrm{Ge} / \mathrm{Ag} / \mathrm{Sb}$ layers. A structural model comprising 20 cation layers and 20 anion layers was built using space group $P$ $\overline{3} m 1$. While fitting the XRD pattern, the layers were initially spaced equally. Refinement of the Te $z$-coordinates was then carried out using a set of soft constraints to keep the ( $\mathrm{Ge} / \mathrm{Ag} /$ $\mathrm{Sb}$ )-Te bond distances within the range 2.8-3.2 $\AA$; the average bond distance in the R-phase is $3.0 \AA$. Due to the large number of parameters, the $\mathrm{Ge} / \mathrm{Ag} / \mathrm{Sb}$ sublattice was kept fixed. It is known that GeTe-based materials can easily accommodate cation vacancies. ${ }^{5}$ Furthermore, weak peaks corresponding to the known phase $\mathrm{Ag}_{8} \mathrm{GeTe}_{6}$ with the argyrodite structure ${ }^{30}$ appeared in the XRD patterns above $200{ }^{\circ} \mathrm{C}$ together with the trigonal phase (Figure $3 \mathrm{~b}$ ). This suggests that a spontaneous net precipitation of cations from the TAGS-85 matrix occurs. We therefore explored the possibility of ordered cation vacancies in the 40-layer trigonal structure and obtained a significant improvement in fit when the cations on the $1 b$ $(0,0,1 / 2)$ site were removed. Our model fits the peak intensities reasonably well (Supporting Information Figure S3a), although the remaining degree of mismatch suggests that it may not be fully accurate. The extensive peak overlap in this mixed-phase sample and the large number of structural parameters prevent further analysis of the current data. Nevertheless, our tentative structure solution is presented in Table 2, and the structure is shown schematically in Figure 3c. We hereafter refer to this structure using the Ramsdell notation $39 \mathrm{P},{ }^{31}$ where $\mathrm{P}$ corresponds to a primitive unit cell. It is rather similar to the 39R structure of $\mathrm{Ge}_{3} \mathrm{SnSb}_{2} \mathrm{Te}_{7}$, which also contains a cubic $\mathrm{ABCABC}$ layer stacking sequence with van der Waals gaps at the missing cation layers. ${ }^{32}$

The unit cell angle of the R-phase gradually increases on heating from room temperature (Figure $4 \mathrm{c}$ ). When the angle reaches $60^{\circ}$ at $380^{\circ} \mathrm{C}$, the unit cell can be described as cubic (C) with space group $F m \overline{3} m$ and $a=6.0293(2) \AA$. The 39P peaks rapidly diminish above $320{ }^{\circ} \mathrm{C}$ and have fully disappeared at $380{ }^{\circ} \mathrm{C}$ along with the $\mathrm{Ag}_{8} \mathrm{GeTe}_{6}$ peaks, implying that the precipitate redissolves into the TAGS-85 phase. In the C-phase, the rhombohedral $211 / 10 \overline{1}$ doublet has become fully merged into a single symmetric peak, indexed as 400 in the cubic cell (Figure 3b).

On subsequent cooling, the $39 \mathrm{P}$ phase reappears at $300{ }^{\circ} \mathrm{C}$ together with the $\mathrm{Ag}_{8} \mathrm{GeTe}_{6}$ precipitate and initially coexists with the C-phase. Splitting of the C-phase 400 peak indicates that the C-phase becomes rhombohedral below $220{ }^{\circ} \mathrm{C}$, and the sample once cooled to room temperature consists of a mixture of $\mathrm{R}, 39 \mathrm{P}$, and $\mathrm{Ag}_{8} \mathrm{GeTe}_{6}$. The presence of $\mathrm{Ag}_{8} \mathrm{GeTe}_{6}$ was confirmed in a thermally cycled sample by high-angle annular dark-field imaging, where precipitates of micron size 


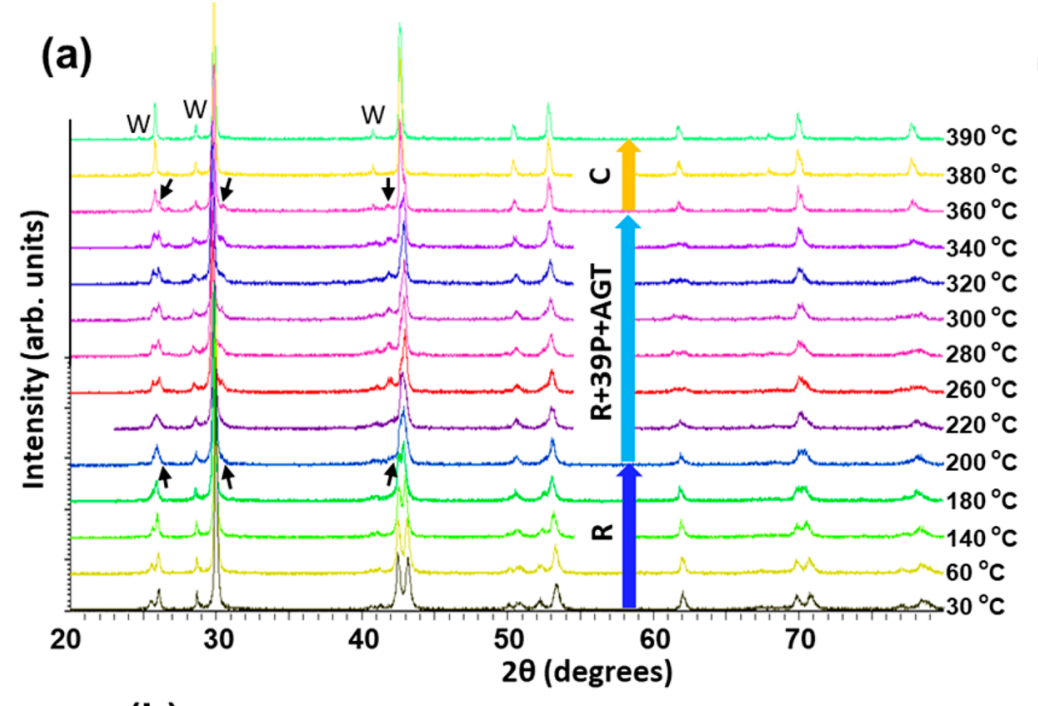

(b)

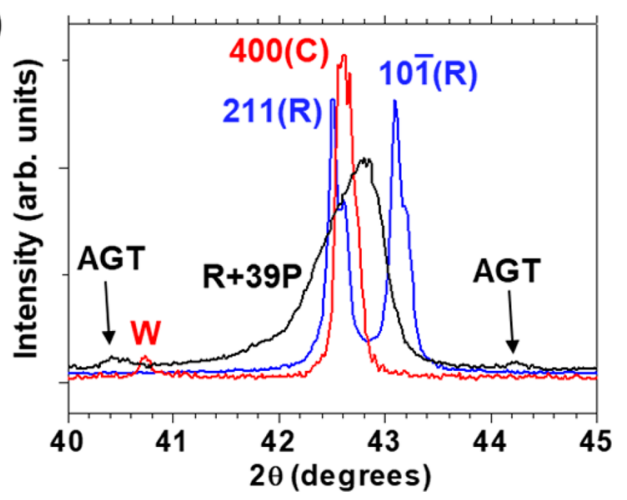

(c)

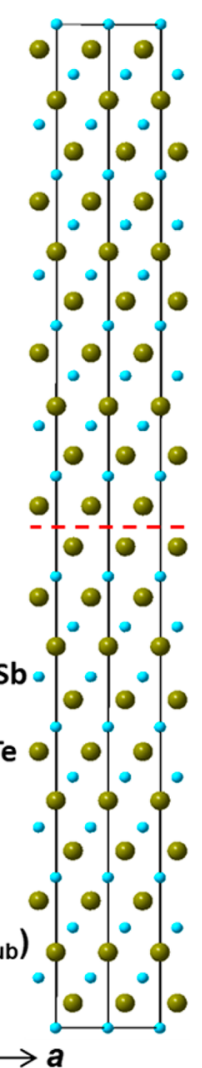

Figure 3. High-temperature structural properties of TAGS-85. (a) XRD patterns collected on initial heating of an as-synthesized single-phase sample. Reflections marked by "W" are assigned to parasitic tungsten $\mathrm{K} \alpha$ radiation from the X-ray tube. Arrows indicate reflections from the 39P phase. (b) Close-up view of the $2 \theta=41-45^{\circ}$ region of XRD patterns of the as-synthesized R-phase measured at $20{ }^{\circ} \mathrm{C}$ (blue), C-phase measured at $400{ }^{\circ} \mathrm{C}$ after initial heating (red), and mixed R $+39 \mathrm{P}+\mathrm{AGT}$ measured at $20^{\circ} \mathrm{C}$ after four heating/cooling cycles. (c) ac-Plane view of $39 \mathrm{P}$ crystal structure showing two unit cells along $a$. The red dashed line indicates a vacant cation layer.

Table 2. Refined Structural Parameters of 39P Phase of TAGS-85 at $280{ }^{\circ} \mathrm{C}^{a}$

\begin{tabular}{|c|c|c|c|c|c|c|}
\hline atom & site & $x$ & $y$ & $z$ & site occupancy & $U_{\text {iso }}\left(\AA^{2}\right)$ \\
\hline $\mathrm{Ge} 1 / \mathrm{Ag} 1 / \mathrm{Sb} 1$ & $1 a$ & 0 & 0 & 0 & $0.7391 / 0.1304 / 0.1304$ & $0.086(5)$ \\
\hline $\mathrm{Te} 2$ & $2 d$ & $1 / 3$ & $2 / 3$ & $0.0250(7)$ & 1 & $0.016(3)$ \\
\hline $\mathrm{Ge} 3 / \mathrm{Ag} 3 / \mathrm{Sb} 3$ & $2 d$ & $2 / 3$ & $1 / 3$ & 0.05 & $0.7391 / 0.1304 / 0.1304$ & $0.086(5)$ \\
\hline Te4 & $2 c$ & 0 & 0 & $0.0756(6)$ & 1 & $0.016(3)$ \\
\hline Ge5/Ag5/Sb5 & $2 d$ & $1 / 3$ & $2 / 3$ & 0.10 & $0.7391 / 0.1304 / 0.1304$ & $0.086(5)$ \\
\hline Te6 & $2 d$ & $2 / 3$ & $1 / 3$ & $0.1268(6)$ & 1 & $0.016(3)$ \\
\hline $\mathrm{Ge} 7 / \mathrm{Ag} 7 / \mathrm{Sb} 7$ & $2 c$ & 0 & 0 & 0.15 & $0.7391 / 0.1304 / 0.1304$ & $0.086(5)$ \\
\hline Te8 & $2 d$ & $1 / 3$ & $2 / 3$ & $0.1766(6)$ & 1 & $0.016(3)$ \\
\hline $\mathrm{Ge} 9 / \mathrm{Ag} 9 / \mathrm{Sb} 9$ & $2 d$ & $2 / 3$ & $1 / 3$ & 0.20 & $0.7391 / 0.1304 / 0.1304$ & $0.086(5)$ \\
\hline Te10 & $2 c$ & 0 & 0 & $0.2267(5)$ & 1 & $0.016(3)$ \\
\hline Ge11/Ag11/Sb11 & $2 d$ & $1 / 3$ & $2 / 3$ & 0.25 & $0.7391 / 0.1304 / 0.1304$ & $0.086(5)$ \\
\hline Te12 & $2 d$ & $2 / 3$ & $1 / 3$ & $0.2755(6)$ & 1 & $0.016(3)$ \\
\hline Ge13/Ag13/Sb13 & $2 c$ & 0 & 0 & 0.30 & $0.7391 / 0.1304 / 0.1304$ & $0.086(5)$ \\
\hline Te14 & $2 d$ & $1 / 3$ & $2 / 3$ & $0.3272(7)$ & 1 & $0.016(3)$ \\
\hline Ge15/Ag15/Sb15 & $2 d$ & $2 / 3$ & $1 / 3$ & 0.35 & $0.7391 / 0.1304 / 0.1304$ & $0.086(5)$ \\
\hline Te16 & $2 c$ & 0 & 0 & $0.3804(5)$ & 1 & $0.016(3)$ \\
\hline Ge17/Ag17/Sb17 & $2 d$ & $1 / 3$ & $2 / 3$ & 0.40 & $0.7391 / 0.1304 / 0.1304$ & $0.086(5)$ \\
\hline Te18 & $2 d$ & $2 / 3$ & $1 / 3$ & $0.4293(7)$ & 1 & $0.016(3)$ \\
\hline Ge19/Ag19/Sb19 & $2 c$ & 0 & 0 & 0.45 & $0.7391 / 0.1304 / 0.1304$ & $0.086(5)$ \\
\hline Te20 & $2 d$ & $1 / 3$ & $2 / 3$ & $0.4798(6)$ & 1 & $0.016(3)$ \\
\hline
\end{tabular}

${ }^{a}$ Space group $P \overline{3} m 1$ (No. 164), $a=4.2333(7) \AA, c=69.895(17) \AA$, wRp $=0.276, R\left(F^{2}\right)=0.210$. 
(a)

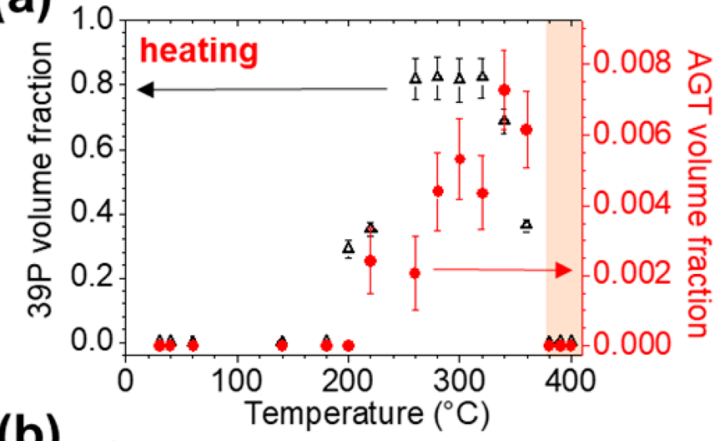

(b)

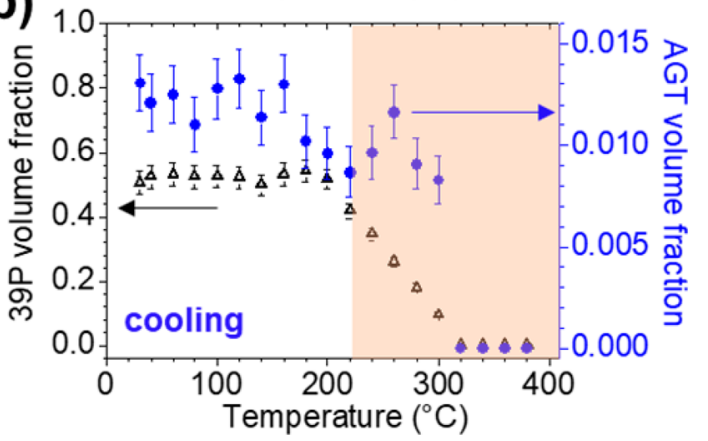

(c)

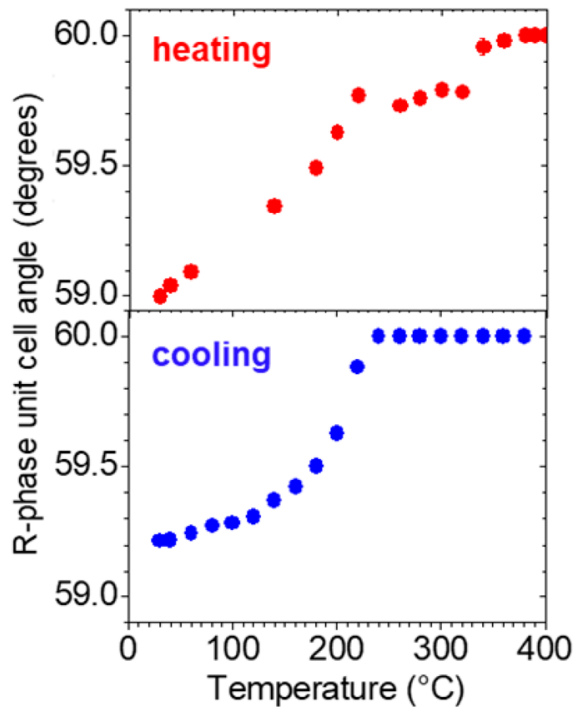

Figure 4. (a, b) Temperature dependence of volume fractions of 39P (black $\triangle$ ) and AGT (red/blue $\bullet$ ) phases on initial heating and subsequent cooling, respectively. The shaded areas of the plots indicate the temperature regions in which $39 \mathrm{P}$ coexists with the C-phase (in which the unit cell angle is $\left.60^{\circ}\right)$. (c) Evolution of unit cell angle of R-phase on initial heating (top) and subsequent cooling (bottom).

were observed (see Supporting Information Figure S3d). The evolution of the refined phase fractions and the R-phase unit cell angle on cooling are plotted in Figure 4b,c, respectively; in these refinements the atomic coordinates of the 39P phase were fixed to the solution obtained at $280{ }^{\circ} \mathrm{C}$ on initial heating. Further thermal cycling reverses these transitions, which take place at $\sim 50{ }^{\circ} \mathrm{C}$ higher on heating than on cooling. However, the pure R-phase is never recovered, and it always coexists with 39P down to room temperature after the initial heating cycle, giving a characteristic broad XRD profile at the position of the $211 / 10 \overline{1}$ doublet (Figure 3b). EDX data were collected on a thermally cycled sample and showed that no significant change in chemical composition had occurred (Supporting Information, Table 2). We also performed neutron diffraction at $20^{\circ} \mathrm{C}$ on a sample that had undergone one heating and cooling cycle and thus contains an approximately $1: 1$ ratio by volume of $\mathrm{R}$ and 39P phases (Figure 4b). The neutron data have lower angular resolution than the XRD data and hence a greater degree of peak overlap; thus, the phase fractions were fixed at those determined from the XRD data collected at $30{ }^{\circ} \mathrm{C}$ after one thermal cycle, and the atomic coordinates were not refined. As shown in the Supporting Information, Figure S3b, the fit is satisfactory, confirming that the $\mathrm{R}+39 \mathrm{P}+\mathrm{Ag}_{8} \mathrm{GeTe}_{6}$ structural model obtained from the XRD data is reasonable. The phase transitions of TAGS-85 that occur on thermal cycling are summarized in Figure 5a.

The evolution of the phase fractions plotted in Figure $4 \mathrm{a}, \mathrm{b}$ suggests that the $39 \mathrm{P}$ and $\mathrm{Ag}_{8} \mathrm{GeTe}_{6}$ phases are closely linked to each other. It is likely that the appearance of the cationdeficient 39P phase is driven by the net precipitation of cations from the TAGS-85 matrix associated with $\mathrm{Ag}_{8} \mathrm{GeTe}_{6}$. We note that $\mathrm{Ag}_{8} \mathrm{GeTe}_{6}$ as a second phase has been observed by TEM and XRD at room temperature in previous studies of TAGS-85 and TAGS-80 samples. ${ }^{12,21}$ Although no correlation with the formation of any layered phase such as 39P was reported, such

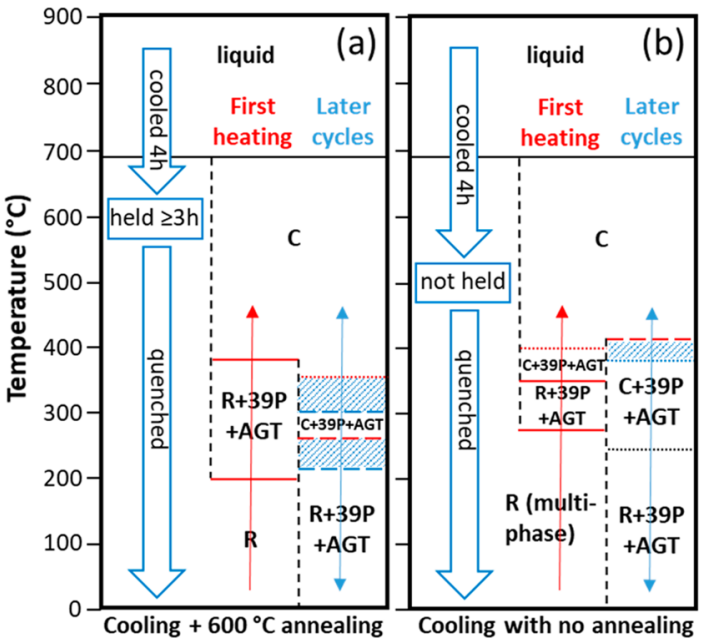

Figure 5. Diagram showing the phase transitions of TAGS- 85 samples cooled from the melt with (a) intermediate annealing step of $\geq 3 \mathrm{~h}$ at $600{ }^{\circ} \mathrm{C}$ and (b) no annealing step. Transitions are shown for the initial heating cycle and subsequent thermal cycling. The labels R, 39P, and $\mathrm{C}$ denote different TAGS-85 polymorphs (see main text for details). AGT denotes the $\mathrm{Ag}_{8} \mathrm{GeTe}_{6}$ impurity phase. Solid horizontal lines indicate phase transition temperatures determined within an uncertainty of $\pm 10{ }^{\circ} \mathrm{C}$, dashed horizontal lines denote borders of a heating/cooling hysteresis region (shaded area) determined within an uncertainty of $\pm 10{ }^{\circ} \mathrm{C}$, and dotted horizontal lines indicate phase transition temperatures or borders of a hysteresis region with a larger uncertainty than $\pm 10^{\circ} \mathrm{C}$ due to lack of data. These horizontal lines are color-coded: red $=$ transition on heating only; blue $=$ transition on cooling only; black $=$ transition on heating and cooling.

a phase could easily be overlooked if the evolution of the whole XRD pattern, including peak shapes, is not examined carefully. Similar layered phases with long $c$-axes have been observed in the $(\mathrm{GeTe})_{n} \mathrm{Sb}_{2} \mathrm{Te}_{3}$ family of materials (commonly known as 

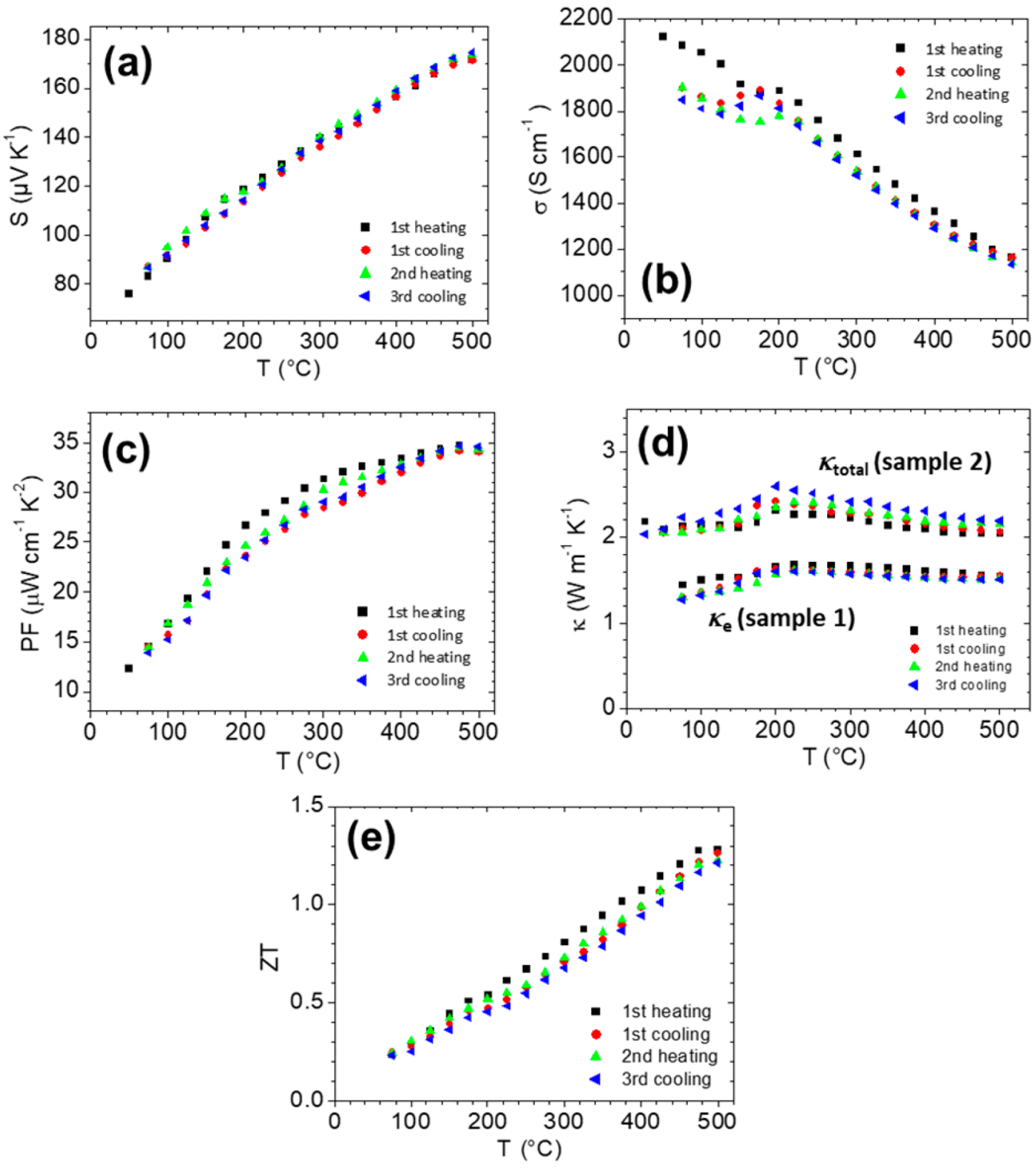

Figure 6. Thermoelectric properties of TAGS-85 over three thermal cycles. (a) Absolute Seebeck coefficient $S$, (b) electrical conductivity $\sigma$, and (c) power factor $(\mathrm{PF})$. (d) Total thermal conductivity $(\kappa)$ of a second sample cut from the same ingot. The electronic thermal conductivity of the first sample is also shown. (e) ZT obtained by combining data from samples 1 and 2.

GST), which are related to TAGS- $x .{ }^{33}$ For example, $\mathrm{Ge}_{2} \mathrm{Sb}_{2} \mathrm{Te}_{5}$, $\mathrm{Ge}_{1} \mathrm{Sb}_{4} \mathrm{Te}_{7}, \mathrm{Ge}_{1} \mathrm{Sb}_{2} \mathrm{Te}_{4}$, and $\mathrm{Ge}_{3} \mathrm{Sb}_{2} \mathrm{Te}_{6}$ adopt trigonal structures consisting of $9,12,21$, and 33 alternating anion and cation layers, respectively. ${ }^{33-35}$ Analogous phase transitions have also been reported for GST materials doped with In, Co, and $\mathrm{Sn}^{9,36,37}$

We also followed the structural evolution of an assynthesized multiphase rhombohedral sample on thermal cycling (Supporting Information, Figure S4). The phase behavior is qualitatively similar to that of the pure R-phase and is summarized in Figure $5 \mathrm{~b}$. The XRD peaks become sharper after the first heating and cooling cycle suggesting that the sample becomes more homogeneous after effectively being annealed, but as discussed below the thermoelectric properties remain inferior.

3. Thermoelectric Properties. The Seebeck coefficient and electrical conductivity of an as-synthesized single-phase rhombohedral sample were measured over three thermal cycles between room temperature and $500{ }^{\circ} \mathrm{C}$ (Figure 6a,b). The thermal diffusivity of a second slice of the same ingot was also measured over three thermal cycles (the thermal conductivity is shown in Figure 6d). Both slices had densities 94-95\% of that calculated from XRD data. The ZT plot shown in Figure 6e was obtained by combining the data from the two slices.

An anomaly in the electrical conductivity and a maximum in the thermal conductivity are apparent at $\sim 200{ }^{\circ} \mathrm{C}$ in the first heating cycle, which corresponds to the initial appearance of the 39P and $\mathrm{Ag}_{8} \mathrm{GeTe}_{6}$ phases (Figure 5a). None of the measured properties show any signature of the second transition at $380{ }^{\circ} \mathrm{C}$ where the gradually increasing unit cell angle of the R-phase reaches $60^{\circ}$ and the 39P and $\mathrm{Ag}_{8} \mathrm{GeTe}_{6}$ phases disappear. However, in subsequent consecutive heating and cooling cycles, hysteresis is observed in the electrical conductivity between 130 and $220{ }^{\circ} \mathrm{C}$. Because the 39P phase and $\mathrm{Ag}_{8} \mathrm{GeTe}_{6}$ appear/disappear at $300{ }^{\circ} \mathrm{C}$ or above, the hysteresis is probably not associated with any phase transition but rather with the rapidly changing unit cell angle of the Rphase in this temperature range (see Figure $3 \mathrm{~d}$ ). The changing rhombohedral distortion will lead to a constantly evolving degree of strain due to lattice mismatch at domain walls, which 
will in turn likely lead to changes in the nanostructure. Domain rearrangement would likely involve a slow, diffusion-controlled process. The Seebeck coefficient increases smoothly over the entire temperature range to reach $\sim 175 \mu \mathrm{V} \mathrm{K}^{-1}$ at $500{ }^{\circ} \mathrm{C}$ and barely changes with thermal cycling. This is on the lower side of the distribution of values in previous reports (see Supporting Information, Table S1). Conversely, the electrical conductivity is considerably higher than in all previous reports, giving a power factor that reaches $0.0035 \mathrm{~W} \mathrm{~m}^{-1} \mathrm{~K}^{-2}$ at $500{ }^{\circ} \mathrm{C}$ (Figure $6 \mathrm{c})$, the highest yet reported for undoped TAGS-85. We checked the reproducibility of our results by measuring other slices of the same ingot. The greatest variability was found in the electrical conductivity; the values in Figure $6 \mathrm{~b}$ can be considered average in this respect, and the power factor of $0.0035 \mathrm{~W} \mathrm{~m}^{-1} \mathrm{~K}^{-2}$ at $500{ }^{\circ} \mathrm{C}$ has a variability of $\pm 0.0007 \mathrm{~W}$ $\mathrm{m}^{-1} \mathrm{~K}^{-2}$ between different slices of the same ingot. The significant differences in electrical conductivity between different samples might be attributable to varying concentrations of microcracks or pores in the samples. Nevertheless, it is clear that annealing at $600{ }^{\circ} \mathrm{C}$ on initial cooling from the melt gives an average power factor among different samples that is significantly higher than all literature reports to date.

The thermal conductivity (Figure 6d) exhibits a broad maximum of up to $\sim 2.6 \mathrm{~W} \mathrm{~m}^{-1} \mathrm{~K}^{-1}$ on both heating and cooling in the vicinity of the $\mathrm{R}-\mathrm{C}$ transition. The thermal conductivity of $\sim 2.1-2.3 \mathrm{~W} \mathrm{~m}^{-1} \mathrm{~K}^{-1}$ at $500{ }^{\circ} \mathrm{C}$ was consistent between different samples and represents a value that is $\sim 25 \%$ higher than in previous reports, which can be attributed to the higher electrical conductivity. The electronic thermal conductivity $\kappa_{\mathrm{e}}$ was calculated for sample 1 from the data in Figure $6 \mathrm{a}, \mathrm{b}$ using the Wiedemann-Franz law $\kappa_{\mathrm{e}} / \sigma=L T$, where the Lorenz number as a function of temperature was obtained from the relationship $L=1.5+\exp (-|S| / 116)$ as proposed by Kim et al. for nondegenerate semiconductors ${ }^{38}$ (Supporting Information, Figure S5). The data plotted in Figure 6d imply that the lattice thermal conductivity $\kappa_{1}$ is in the range $0.7-1.0 \mathrm{~W} \mathrm{~m}^{-1}$ $\mathrm{K}^{-1}$, in agreement with that reported by $\mathrm{Zhu}$ et al. ${ }^{17}$ It is noticeable that $\kappa_{\text {total }}$ is slightly lower on initial heating, despite $\sigma$ being higher. This implies a significant increase in $\kappa_{1}$ after the first heating cycle, which is likely due to a change in domain structure and requires further investigation. We also note that, as in previous reports, $\kappa_{\text {total }}$ does not increase on entering the cubic regime above $400{ }^{\circ} \mathrm{C}$. Here only antiphase domain boundaries should remain if the symmetry is truly cubic. However, previous studies of GeTe using local X-ray and neutron scattering techniques have shown that the Peierls distortion remains in the high-temperature cubic phase, with domain sizes of the order of two unit cells. ${ }^{20,39}$ This might also be the case in TAGS-85 and explain why $\kappa_{\text {total }}$ remains low in the C-phase.

The good power factors of our samples are somewhat countered by the relatively high thermal conductivity, leading to a dimensionless figure of merit ZT of $\sim 1.3$ at $500{ }^{\circ} \mathrm{C}$ that is robust with respect to thermal cycling (Figure 6e). This is similar to the ZT values reported for samples in the literature with lower electrical conductivity (see Supporting Information, Table S1). We note that we have made no attempt to artificially nanostructure our samples, as for example could be attempted by spark plasma sintering. Therefore, there is likely to be scope for increasing ZT significantly.

The temperature dependent thermoelectric properties of a multiphase sample are shown in the Supporting Information, Figure S6. The Seebeck coefficient and electrical conductivity are $\sim 10 \%$ lower over most of the temperature range, probably due to the structural inhomogeneity of this sample. This leads to a power factor of $0.0024 \mathrm{~W} \mathrm{~m}^{-1} \mathrm{~K}^{-2}$ at $400{ }^{\circ} \mathrm{C}$ that does not improve on subsequent thermal cycling, which is lower than most literature reports on TAGS-85 (Supporting Information, Table S1). The thermal conductivity is $\sim 2.0 \mathrm{~W} \mathrm{~m}^{-1} \mathrm{~K}^{-1}$ after the initial heating cycle, which is slightly greater than the values of $1.4-1.9 \mathrm{~W} \mathrm{~m}^{-1} \mathrm{~K}^{-1}$ reported in the literature because the electrical conductivity remains relatively high. Therefore, the $\mathrm{ZT}$ value from the combined data is rather low, reaching 0.8 at $400{ }^{\circ} \mathrm{C}$. This result illustrates the importance of good structural homogeneity for the thermoelectric performance of TAGS-85, which can be ensured by annealing at $600{ }^{\circ} \mathrm{C}$ on initial cooling from the melt.

\section{CONCLUSIONS}

We find that the phase transitions that occur in TAGS- 85 with temperature are much more complex than previously thought. The room-temperature rhombohedral phase is partially transformed to a new trigonal structure containing ordered layers of cation vacancies above $200{ }^{\circ} \mathrm{C}$. This occurs together with the spontaneous precipitation of $1-2 \%$ by volume of the cationrich phase $\mathrm{Ag}_{8} \mathrm{GeTe}_{6}$. The previously reported high-temperature cubic polymorph is reached above $380{ }^{\circ} \mathrm{C}$, where $\mathrm{Ag}_{8} \mathrm{GeTe}_{6}$ redissolves in the TAGS-85 matrix. On subsequent cooling the trigonal layered phase and the $\mathrm{Ag}_{8} \mathrm{GeTe}_{6}$ precipitate reappear and coexist with the rhombohedral polymorph down to room temperature. The trigonal phase and the $\mathrm{Ag}_{8} \mathrm{GeTe}_{6}$ precipitates are observed in all the samples that we have investigated and therefore cannot be responsible for the variability in thermoelectric properties that we find between our own TAGS-85 samples and those in the literature. However, the question of how the trigonal phase influences the microstructure/nanostructure of TAGS-85 and hence the lattice thermal conductivity as a function of temperature is an interesting question that should be investigated in future studies.

We also show that an annealing step at $600{ }^{\circ} \mathrm{C}$ during the initial cooling procedure from the melt is essential to obtain single-phase rhombohedral samples of TAGS-85. The electrical conductivity of these homogeneous, annealed samples is considerably higher than in all previous reports on TAGS- 85 . There is no detrimental effect on the Seebeck coefficient, which results in a record high power factor for undoped TAGS-85 of $0.0035 \mathrm{~W} \mathrm{~m}^{-1} \mathrm{~K}^{-2}$ at $500{ }^{\circ} \mathrm{C}$ that is maintained on repeated thermal cycling. The electronic thermal conductivity is also increased, giving a $\mathrm{ZT}$ of $\sim 1.3$ at $500{ }^{\circ} \mathrm{C}$ which is in line with the best literature values. Faster cooling procedures give inhomogeneous samples containing multiple rhombohedral phases with a distribution of lattice parameters. These samples exhibit inferior Seebeck coefficients and electric conductivity, with $\mathrm{ZT}$ values consequently $<1.0$. Our work thus provides a guide to maximize the power factor of TAGS-85 by ensuring good structural coherence, which might be further increased by doping. Furthermore, artificial nanostructuring of these high power factor samples should lead to even better thermoelectric performance.

\section{ASSOCIATED CONTENT}

Supporting Information

The Supporting Information is available free of charge on the ACS Publications website at DOI: 10.1021/acs.inorgchem.7b02433. 
Comparison of thermoelectric properties of TAGS-85 literature samples, chemical composition analysis of samples, fitted XRD and neutron diffraction patterns of as-prepared and thermally cycled samples, relationship between rhombohedral and hexagonal/trigonal settings of the R unit cell, TEM and SAED images of thermally cycled sample, high-temperature XRD patterns of multiphase sample, and thermoelectric properties of multiphase sample (PDF)

\section{AUTHOR INFORMATION}

\section{Corresponding Author \\ *E-mail: g.r.blake@rug.nl. \\ ORCID $\odot$}

Paul A. Vermeulen: 0000-0003-2035-3383

Oliver Oeckler: 0000-0003-0149-7066

Graeme R. Blake: 0000-0001-9531-7649

\section{Author Contributions}

The manuscript was written through contributions of all authors. All authors have given approval to the final version of the manuscript.

\section{Notes}

The authors declare no competing financial interest.

\section{ACKNOWLEDGMENTS}

The authors thank J. Baas for technical support. Financial support by the Studienstiftung des deutschen Volkes (fellowship for S.S.) is gratefully acknowledged.

\section{REFERENCES}

(1) Snyder, G. J.; Toberer, E. S. Complex Thermoelectric Materials. Nat. Mater. 2008, 7, 105-114.

(2) Sootsman, J. R.; Chung, D. Y.; Kanatzidis, M. G. New and Old Concepts in Thermoelectric Materials. Angew. Chem., Int. Ed. 2009, 48, 8616-8639.

(3) Kanatzidis, M. G. Nanostructured Thermoelectrics: The New Paradigm? Chem. Mater. 2010, 22, 648-659.

(4) Zhao, L.-D; Dravid, V. P.; Kanatzidis, M. G. The Panoscopic Approach to High Performance Thermoelectrics. Energy Environ. Sci. 2014, 7, 251-268.

(5) Levin, E. M.; Besser, M. F.; Hanus, R. Electronic and Thermal Transport in GeTe: A Versatile Base for Thermoelectric Materials. J. Appl. Phys. 2013, 114, 083713.

(6) Damon, D. H.; Lubell, M. S.; Mazelsky, R. M. Nature of the Defects in Germanium Telluride. J. Phys. Chem. Solids 1967, 28, 520522.

(7) Perumal, S.; Roychowdhury, S.; Biswas, K. High performance thermoelectric materials and devices based on GeTe. J. Mater. Chem. C 2016, 4, 7520-7536.

(8) Lee, H. S.; Kim, B.-S.; Cho, C.-W.; Oh, M.-W.; Min, B.-K.; Park, S.-D.; Lee, H.-W. Herringbone Structure in GeTe-Based Thermoelectric Materials. Acta Mater. 2015, 91, 83-90.

(9) Fahrnbauer, F.; Souchay, D.; Wagner, G.; Oeckler, O. High Thermoelectric Figure of Merit Values of Germanium Antimony Tellurides with Kinetically Stable Cobalt Germanide Precipitates. J. Am. Chem. Soc. 2015, 137, 12633-12638.

(10) Perumal, S.; Roychowdhury, S.; Negi, D. S.; Datta, R.; Biswas, K. High thermoelectric performance and enhanced mechanical stability of p-type $\mathrm{Ge}_{1-\mathrm{x}} \mathrm{Sb}_{\mathrm{x}}$ Te. Chem. Mater. 2015, 27, 7171-7178.

(11) Chen, Y.; Jaworski, C. M.; Gao, Y. B.; Wang, H.; Zhu, T. J.; Snyder, G. J.; Heremans, J. P.; Zhao, X. B. Transport Properties and Valence Band Feature of High-Performance $(\mathrm{GeTe})_{85}\left(\mathrm{AgSbTe}_{2}\right)_{15}$ Thermoelectric Materials. New J. Phys. 2014, 16, 013057.
(12) Davidow, J.; Gelbstein, Y. A Comparison Between the Mechanical and Thermoelectric Properties of Three Highly Efficient p-Type GeTe-Rich Compositions: TAGS-80, TAGS-85, and 3\% $\mathrm{Bi}_{2} \mathrm{Te}_{3}$-Doped $\mathrm{Ge}_{0.87} \mathrm{~Pb}_{0.13}$ Te. J. Electron. Mater. 2013, 42, 1542-1549.

(13) Thompson, A. J.; Sharp, J. W.; Rawn, C. J. Microstructure and Crystal Structure in TAGS Compositions. J. Electron. Mater. 2009, 38, 1407-1411.

(14) Levin, E. M.; Cook, B. A.; Harringa, J. L.; Bud'ko, S. L.; Venkatasubramanian, R.; Schmidt-Rohr, K. Analysis of Ce- and YbDoped TAGS-85 Materials with Enhanced Thermoelectric Figure of Merit. Adv. Funct. Mater. 2011, 21, 441-447.

(15) Levin, E. M.; Bud'ko, S. L.; Schmidt-Rohr, K. Enhancement of Thermopower of TAGS-85 High-Performance Thermoelectric Material by Doping with the Rare Earth Dy. Adv. Funct. Mater. 2012, 22, 2766-2774.

(16) Schröder, T.; Rosenthal, T.; Giesbrecht, N.; Nentwig, M.; Maier, S.; Wang, H.; Snyder, G. J.; Oeckler, O. Nanostructures in Te/Sb/Ge/ Ag (TAGS) Thermoelectric Materials Induced by Phase Transitions Associated with Vacancy Ordering. Inorg. Chem. 2014, 53, 7722-7729.

(17) Zhu, T.; Gao, H.; Chen, Y.; Zhao, X. Ioffe-Regel Limit and Lattice Thermal Conductivity Reduction of High Performance $\left(\mathrm{AgSbTe}_{2}\right)_{15}(\mathrm{GeTe})_{85}$ Thermoelectric Materials. J. Mater. Chem. A 2014, 2, 3251-3256.

(18) Cook, B. A.; Kramer, M. J.; Wei, X.; Harringa, J. L.; Levin, E. M. Nature of the Cubic to Rhombohedral Structural Transformation in $\left(\mathrm{AgSbTe}_{2}\right)_{15}(\mathrm{GeTe})_{85}$ Thermoelectric Material. J. Appl. Phys. 2007, 101, 053715.

(19) Cook, B. A.; Wei, X.; Harringa, J. L.; Kramer, M. J. In-situ Elevated-Temperature TEM Study of $\left(\mathrm{AgSbTe}_{2}\right)_{15}(\mathrm{GeTe})_{85}$. J. Mater. Sci. 2007, 42, 7643-7646.

(20) Salvador, J. R.; Yang, J.; Shi, X.; Wang, H.; Wereszczak, A. A. Transport and Mechanical Property Evaluation of (AgSb$\mathrm{Te})_{1-\mathrm{x}}(\mathrm{GeTe})_{\mathrm{x}}(\mathrm{x}=0.80,0.82,0.85,0.87,0.90)$. J. Solid State Chem. 2009, 182, 2088-2095.

(21) Yang, S. H.; Zhu, T. J.; Zhang, S. N.; Shen, J. J.; Zhao, X. B. Natural Microstructure and Thermoelectric Performance of (Ge$\mathrm{Te})_{80}\left(\mathrm{Ag}_{\mathrm{y}} \mathrm{Sb}_{2-\mathrm{y}} \mathrm{Te}_{3-\mathrm{y}}\right)_{20}$. J. Electron. Mater. 2010, 39, 2127-2131.

(22) van Eijck, L.; Cussen, L. D.; Sykora, G. J.; Schooneveld, E. M.; Rhodes, N. J.; van Well, A. A.; Pappas, C. Design and Performance of a Novel Neutron Powder Diffractometer: PEARL at TU Delft. J. Appl. Crystallogr. 2016, 49, 1398-1401.

(23) Larson, A. C.; von Dreele, R. B. General Structure Analysis System (GSAS), Los Alamos National Laboratory LAUR Report; 2004, No. 86748.

(24) Dusza, L. Combined Solution of the Simultaneous Heat Loss and Finite Pulse Corrections with the Laser Flash Method. High Temp. - High Pressures 1995, 27/28, 467-473.

(25) Wang, H.; Porter, W. D.; Böttner, H.; König, J.; Chen, L.; Bai, S.; Tritt, T. M.; Mayolet, A.; Senawiratne, J.; Smith, C.; Harris, F.; Gilbert, P.; Sharp, J.; Lo, J.; Kleinke, H.; Kiss, L. Transport properties of bulk thermoelectrics: an international round-robin study, part II: thermal diffusivity, specific heat, and thermal conductivity. J. Electron. Mater. 2013, 42, 1073-1084.

(26) Stephens, P. W. Phenomenological Model of Anisotropic Peak Broadening in Powder Diffraction. J. Appl. Crystallogr. 1999, 32, 281289.

(27) Yang, S. H.; Zhu, T. J.; Sun, T.; He, J.; Zhang, S. N.; Zhao, X. B. Nanostructures in High-Performance $(\mathrm{GeTe})_{\mathbf{x}}\left(\mathrm{AgSbTe}_{2}\right)_{100-\mathrm{x}}$ Thermoelectric Materials. Nanotechnology 2008, 19, 245707.

(28) Vermeulen, P.; Kumar, A.; ten Brink, G.; Blake, G. R.; Kooi, B. J. Unravelling the Domain Structures in GeTe and $\mathrm{LaAlO}_{3}$. Cryst. Growth Des. 2016, 16, 5915-5922.

(29) Fons, P.; Kolobov, A. V.; Krbal, M.; Tominaga, J.; Andrikopoulos, K. S.; Yannopoulos, S. N.; Voyiatzis, G. A.; Uruga, T. Phase Transition in Crystalline GeTe: Pitfalls of Averaging Effects. Phys. Rev. B: Condens. Matter Mater. Phys. 2010, 82, 155209.

(30) von Unterrichter, J.; Range, K. J. $\mathrm{Ag}_{8} \mathrm{GeTe}_{6}$, a Representative of the Argyrodite Family. Z. Naturforsch., B: J. Chem. Sci. 1978, 33, 866872. 
(31) Ramsdell, L. S. Studies on Silicon Carbide. Am. Mineral. 1947, 32, 64-82.

(32) Rosenthal, T.; Welzmiller, S.; Neudert, L.; Urban, P.; Fitch, A.; Oeckler, O. Novel Superstructure of the Rocksalt Type and Element Distribution in Germanium Tin Antimony Tellurides. J. Solid State Chem. 2014, 219, 108-117.

(33) Siegrist, T.; Merkelbach, P.; Wuttig, M. Phase Change Materials: Challenges on the Path to a Universal Storage Device. Annu. Rev. Condens. Matter Phys. 2012, 3, 215-237.

(34) Rosenthal, T.; Schneider, M. N.; Stiewe, C.; Döblinger, M.; Oeckler, O. Real Structure and Thermoelectric Properties of GeTeRich Germanium Antimony Tellurides. Chem. Mater. 2011, 23, 43494356.

(35) Karpinsky, O. G.; Shelimova, L. E.; Kretova, M. A.; Fleurial, J.-P. An X-ray Study of the Mixed-Layered Compounds of (Ge$\mathrm{Te})_{\mathrm{n}}\left(\mathrm{Sb}_{2} \mathrm{Te}_{3}\right)_{\mathrm{m}}$ Homologous Series. J. Alloys Compd. 1998, 268, $112-117$.

(36) Fahrnbauer, F.; Urban, P.; Welzmiller, S.; Schröder, T.; Rosenthal, T.; Oeckler, O. $(\mathrm{GeTe})_{\mathrm{n}} \mathrm{SbInTe}_{3}(n \leq 3)$ - Element Distribution and Thermal Behavior. J. Solid State Chem. 2013, 208, 20-26.

(37) Rosenthal, T.; Neudert, L.; Ganter, P.; de Boor, J.; Stiewe, C.; Oeckler, O. Nanostructured Rocksalt-Type Solid Solution Series $\left(\mathrm{Ge}_{1-\mathrm{x}} \mathrm{Sn}_{\mathrm{x}} \mathrm{Te}\right)_{\mathrm{n}} \mathrm{Sb}_{2} \mathrm{Te}_{3}(\mathrm{n}=4,7,12 ; 0 \leq x \leq 1)$ : Thermal Behavior and Thermoelectric Properties. J. Solid State Chem. 2014, 215, 231-240.

(38) Kim, H.-S.; Gibbs, Z. M.; Tang, Y.; Wang, H.; Snyder, G. J. Characterization of Lorenz number with Seebeck coefficient measurement. APL Mater. 2015, 3, 041506.

(39) Matsunaga, T.; Fons, P.; Kolobov, A. V.; Tominaga, J.; Yamada, $\mathrm{N}$. The Order-Disorder Transition in GeTe: Views from Different Length-Scales. Appl. Phys. Lett. 2011, 99, 231907. 